\title{
A On the Use and Abuse of Spatial Instruments
}

\author{
Timm Betz, Scott J. Cook and Florian M. Hollenbach
}

Assistant Professor of Political Science, Department of Political Science, Texas A\&M University, College Station, TX 77843, USA.Email: timm.betz@tamu.edu, sjcook@tamu.edu, fhollenbach@tamu.edu,

URL: www.people.tamu.edu/ timm.betz, scottjcook.net, fhollenbach.org

\begin{abstract}
Instruments based on realizations of the endogenous variable in other units-for instance, regional or global weighted averages-are commonly used in political science. Such spatial instruments have proved attractive: they are convenient to obtain, typically have power, and are plausibly exogenous. We argue that the assumptions underlying spatial instruments remain poorly understood and challenge whether spatial instruments can satisfy the conditions required for valid instruments. First, when cross-unit dependence exists in the endogenous predictor, other cross-unit relationships-spillovers and interdependence-likely exist as well and risk violations of the exclusion restriction. Second, spatial instruments produce simultaneity in the first-stage equation, as left-hand side outcomes are included as right-hand side predictors. Because the instrument and the endogenous variable are simultaneously determined, the exclusion restriction is, necessarily and by construction, violated. Taken together, these concerns lead us to conclude that spatial instruments are rarely, if ever, valid.
\end{abstract}

Keywords: instrumental variables, two-stage least squares, spatial autocorrelation

Faced with concerns of endogeneity, researchers frequently turn to instrumental variable (IV) models. Convenient instruments are often constructed from realizations of the endogenous variable in geographically or otherwise proximate units-thus creating spatial instruments, such as regional or global averages, by using other-unit values as instruments for own-unit endogenous predictors. Following the lead of prominent work in political science and economics, such instruments are increasingly widely used (see, e.g., Stasavage 2005; Acemoglu et al. 2008; Büthe and Milner 2008; Boix 2011). In this research note, we highlight two problems with this strategy. Jointly, they cast substantial doubt on the validity of spatial instruments.

First, spatial instruments require supporting the presence of one type of spatial relationship while concurrently denying other spatial relationships in the form of spillovers in predictors and interdependence in the outcome. ${ }^{1}$ These alternative pathways are rarely discussed explicitly, yet are likely to exist in many applications. When present, they cause violations of the exclusion restriction.

Second, spatial instruments produce simultaneity in the first stage and therefore are not exogenous-put simply, spatial instruments imply a first stage where left-hand side outcomes are included as right-hand side predictors. Despite the wide use of spatial instruments, this has not been acknowledged as an inherent concern in IV estimation. And while the first problem is a matter of plausibility, theory, and evidence, the second problem is not: the violation of the exclusion restriction arises by construction and is endemic to spatial instruments.

Political Analysis (2018)

vol. 26:474-479

DOI: 10.1017/pan.2018.10

Published

21 May 2018

Corresponding author

Scott J. Cook

Edited by

Jens Hainmueller

(c) The Author(s) 2018. Published by Cambridge University Press on behalf of the Society for Political Methodology.
Authors' note: Thanks to Vincent Arel-Bundock and Michael Ward for their helpful comments. All remaining errors are ours alone. Authors are listed in alphabetical order, equal authorship is implied. Replication files for results presented in the Online Appendix are available as Betz, Cook, and Hollenbach (2017) at doi:10.7910/DVN/W4TXDU.

1 There may also be spatial correlation among the disturbances. Given that our concern is primarily bias, we devote little attention to spatial-error models. 


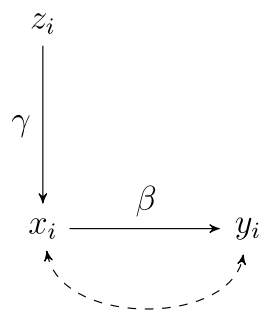

Figure 1. Standard IV relationships where $z_{i}$ is a valid instrument for $x_{i}: x_{i}$ and $y_{i}$ are correlated through unobserved errors; $z_{i}$ causes $x_{i}$, and has no direct effect on $y_{i}$ except through $x_{i}$.

\section{Spatial Instruments}

To clarify the problems with spatial instruments, we consider a simple linear-additive model

$$
y_{i}=\beta x_{i}+e_{i}
$$

where $i=1,2, \ldots, N$ indexes observations, $y_{i}$ is the outcome, $x_{i}$ the predictor, and $e_{i}$ the error term. The ordinary least squares (OLS) estimator of $\beta$ is the ratio of the sample covariance of $x$ and $y$ to the sample variance of $x$, which yields the probability limit

$$
\operatorname{plim}_{n \rightarrow \infty} \widehat{\beta}_{O L S}=\beta+\underbrace{\frac{\operatorname{cov}(x, e)}{\operatorname{var}(x)}}_{\text {endogeneity bias }} .
$$

Equation (2) shows that $\widehat{\beta}_{O L S}$ is asymptotically biased if $\operatorname{cov}(x, e) \neq 0$, that is, if $x$ is endogenous. This result is covered in any introductory econometrics textbook alongside common sources of endogeneity: omitted variable bias, simultaneity, and measurement error.

To address the resulting bias, IV methods are often employed. Following Sovey and Green (2011), we introduce IV estimation using the following system of equations:

$$
\begin{aligned}
& y_{i}=\beta x_{i}+e_{i}, \\
& x_{i}=\gamma z_{i}+u_{i},
\end{aligned}
$$

where $x$ is endogenous if $\operatorname{cov}(u, e) \neq 0 .{ }^{2}$ The variable $z$ is a valid instrument for $x$ if the instrument is relevant, such that $\operatorname{cov}(z, x) \neq 0$, and if it satisfies the exclusion restriction, such that $\operatorname{cov}(z, e)=0$. That is, $z$ needs to be correlated with the endogenous variable $x$, but uncorrelated with the error term in equation (3). Figure 1 depicts the relationships assumed under IV estimation, with no pathways connecting $z$ to $y$ but through $x$.

Given the difficulty of finding valid instruments, researchers frequently turn to values of the endogenous variable from other units as instruments for own-unit endogenous values. More explicitly, spatial instruments are generated as a function of other-unit values, such that $z_{i}=$ $\Sigma_{j \neq i} w_{i, j} x_{j}$, with $w_{i, j}$ indicating the relationship between units $i$ and $j$. In studies on the effect of democracy on growth, for example, neighboring democracy (Persson and Tabellini 2009) or regional waves of democracy (Acemoglu et al. forthcoming) have served as instruments. In both instances, a weighted sum of other-unit democracy scores is used to instrument for own-unit democracy. Spatial instruments are not unique to this literature and have become

2 Alternatively, one could rely on the potential outcomes framework (Angrist, Imbens, and Rubin 1996). Nevertheless, we believe the graph based and structural equation approaches work well together to clarify our argument, and they seem to be more common in studies using spatial instruments. 

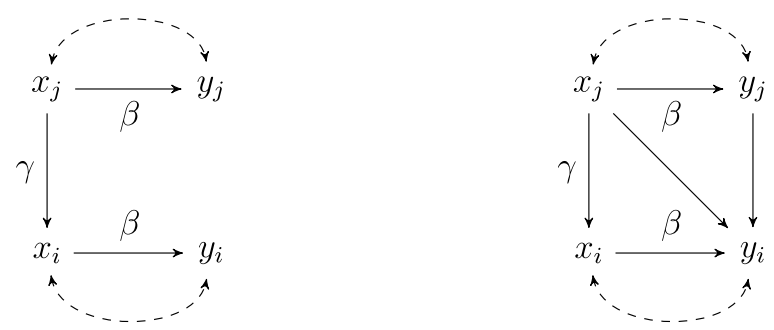

Figure 2. Spatial IV relationships. The left panel shows the assumed model when using spatial instruments: $x_{i}$ is affected by other units $x_{j}$, which serve as instruments. The right panel shows two possible additional spatial relationships that have to be ruled out explicitly: spillovers (from $x_{j}$ to $y_{i}$ ) and interdependence in the outcome (from $y_{j}$ to $y_{i}$ ). Both pathways result in violations of the exclusion restriction.

increasingly popular. Since 2004, over twenty articles have appeared in the APSR, AJPS, JOP, BJPS, and $\mathrm{IO}$ that rely on spatial instruments, and their use seems to have become more common over time. $^{3}$

Spatial instruments are attractive for at least three reasons. First, they are convenient, because the data already exist in the sample. Researchers need only determine an appropriate weighting scheme to construct the instrument-which might be as simple as calculating a regional or global average. Second, the existing literature often provides reasons, such as diffusion, competitive or peer effects, spillovers, or learning, to expect the instrument to have power. Third, it is argued or assumed that such instruments satisfy the exclusion restriction.

In what follows we challenge whether this latter belief-that instruments based on values of the endogenous variable in other units satisfy the exclusion restriction-is plausible (Section 1.1) or, in fact, even possible (Section 1.2). ${ }^{4}$

\subsection{Direct and indirect effects of other-unit predictors}

To highlight the implicit assumptions made when using spatial instruments, we re-express Figure 1 in the left panel of Figure 2, with $x_{j}$, other-unit values of $x_{i}$, inserted for instrument $z_{i}$. Analogously to unit $i$, other units are related to their own outcomes $y_{j}$. As usual with causal path diagrams, note the assumed and implied absence of causal relationships, two of which we have highlighted in the right panel. The right panel displays the same graph, but includes two additional plausible pathways between the instrument and the outcome: directly via spillovers $\left(x_{j} \rightarrow y_{i}\right)$ and indirectly via interdependent outcomes $\left(x_{j} \rightarrow y_{j} \rightarrow y_{i}\right)$. For instruments to be valid, both of these pathways must be ruled out explicitly. This is more difficult to do in the context of spatial instruments, where the argument implies that interdependence exists in one variable but not in others.

First, spillovers in $x$ produce an obvious violation of the exclusion restriction. If other-unit predictor values directly affect outcomes, they cannot be valid instruments. Direct spillovers are often expressed as $y_{i}=\beta x_{i}+\rho \sum_{j \neq i} w_{i, j} x_{j}+e_{i}$, which immediately shows that the exclusion restriction is violated, because the outcome $y_{i}$ is a direct function of $x_{j}$. Spillovers are common and occur when actions, choices, or policies of one unit directly affect outcomes in other units (Manski 1993). For example, unit $j$ 's policies on industrial emissions affect pollution not just in $j$ but also in $i$, as pollutants spread via air and water currents. Similarly, import tariffs in unit $j$ directly affect GDP growth in units $i$ which export that product. In short, spillovers exist whenever externalities are generated by a change in $x_{i}$, and without recourse render spatial instruments invalid.

3 We list the articles in the Online Appendix (Betz, Cook, and Hollenbach 2017). The use of spatial instruments is positively correlated $(\rho=0.51)$ with a time trend.

4 We provide Monte Carlo simulations and an empirical application in the Online Appendix. 
Second, the exclusion restriction is violated through interdependence in the outcome, such that the outcomes of some units affect the outcomes of other units. Theories of interdependence are ubiquitous in political science. The contagion of conflict and crises, the diffusion of policies, the spread of institutions and ideologies, deepening economic integration and resulting policy coordination all provide examples (Franzese and Hays 2007). Interdependence in outcomes entails a relationship between $x_{j}$ and $y_{i}$ through $y_{j}$, and therefore a violation of the exclusion restriction. Interdependence is commonly interpreted as a spatial-autoregressive process, such that $y_{i}=\rho \sum_{j \neq i} w_{i, j} y_{j}+e_{i}$, which can be rewritten as $y_{i}=\rho \sum_{j \neq i} w_{i, j}\left[\beta x_{j}+e_{j}\right]+\beta x_{i}+e_{i}$, indicating how $x_{j}$ affects $y_{i}$. The problem is readily visible in Figure 2. If interdependence exists in the outcomes, then $y_{i}$ is a function of outcomes in other units, $y_{j}$, and therefore of $x_{j}$.

As an illustration, consider the literature on economic development and democratization. In an effort to isolate the causal effect of income on democracy, Acemoglu et al. (2008) use trade-weighted world income to instrument for income. Both spillovers and interdependence in outcomes plausibly occur in this example, rendering the instrument invalid. First, as argued in the literature on economic benchmarking, cross-national income comparisons condition citizen's political actions (Kayser and Peress 2012), which creates spillovers: the benchmarking argument implies that income in other states directly influences political outcomes, such as demands for political liberalization, and therefore violates the exclusion restriction. Second, a vast literature examines the diffusion of democracies (Gleditsch and Ward 2006), which creates interdependence in the outcomes and a violation of the exclusion restriction.

When spatial instruments are used, these additional pathways have to be ruled out explicitly. Figure 2 visually illustrates the difficulty of arguing for the absence of a relationship between $y_{j}$ and $y_{i}$ as well as between $x_{j}$ and $y_{i}$ when an analogous, parallel relationship is argued to exist between $x_{i}$ and $x_{j} .{ }^{5}$ Where spatial relationships exist in one variable, other variables are likely to exhibit spatial relationships as well.

The assumptions necessary for spatial instruments therefore produce a tension. Spatial dependence has to exist in the endogenous variable, while at the same time other spatial relationships must be ruled out. This is rarely done explicitly in applications, and we believe that for most cases it is difficult to convincingly make such an argument: Given that the use of spatial instruments depends on and argues for spatial relationships in the endogenous variable, it is difficult to also argue for the absence of other spatial relationships. ${ }^{6}$

\subsection{Spatial simultaneity and instrumental variables}

Even assuming the conditions elaborated in Section 1.1 are satisfied, a heretofore unrecognized problem with spatial instruments remains: simultaneity between the instrument and endogenous predictor. Intuitively, if units are related to each other, as implied by spatial instruments, unit $i$ is affected by other units, but other units are also affected by unit $i-$ and, in particular, are a function of the endogenous component of unit $i$. Thus, other units cannot serve as valid instruments. A valid instrument needs to provide exogenous variation from outside the system of equations; a spatial instrument cannot satisfy this requirement because it is determined within that system.

The first stage of two-stage least squares (2SLS) is an OLS regression of the endogenous variable on the instrument (and all other exogenous predictors). With spatial instruments, this

5 Equivalently, if the covariance between $x_{i}$ and $x_{j}$ is produced by common shocks in unobservables across units (which creates correlation between units), it requires strong assumptions for this to be true for $x$ but not for $y$.

6 A similar concern exists when using time lags as instruments. Yet, even where researchers recognize the challenges of time-lagged instruments, they fail to see the equivalent issues arising from spatial instruments. For example, Ansell (2008, p. 301) notes that "there are a number of serious problems with using [time] lags as instruments, the chief being that any serial correlation in the error term will retain the bias," and instead advocates using "the average polity score in a country's region as the instrument" for a country's polity score. 
implies

$$
x_{i}=\gamma \Sigma_{j \neq i} w_{i, j} x_{j}+u_{i},
$$

with $w_{i, j}$ indicating the relationship between units $i$ and $j$. Equation (5) can be expressed as

$$
\mathbf{x}=\gamma \mathbf{W} \mathbf{x}+\mathbf{u}
$$

where $\mathbf{W}$ is a $N$-by- $N$ connectivity matrix (with zero elements on the diagonal) defining which and to what extent units are related to each other. Recognizing that the first stage takes the form in equations (5) and (6) makes the problem immediately obvious: because $x$ appears on both the left-hand side and the right-hand side of the equation, the first stage suffers from simultaneity bias. ${ }^{7}$ In particular, this first stage is a spatial-autoregressive model, where it is well known that Wx is not exogenous to, but simultaneously determined with $\mathbf{x}$ (Franzese and Hays 2007). This (spatial) simultaneity necessarily produces a violation of the exclusion restriction: feedback from the endogenous predictor to the instrument makes the instrument a function of the source of endogeneity-that is, it makes the instrument itself endogenous. ${ }^{8}$

The problem arising from simultaneity can also be seen by recalling the standard IV model in equations (3) and (4) and supposing that additionally, $z_{i}=\alpha x_{i}+v_{i}$. Simultaneity between $x$ and $z$ arises if $\alpha \neq 0$. Substitution for $x_{i}$ produces $z_{i}=\alpha\left(\gamma z_{i}+u_{i}\right)+v_{i}$, showing that $z$ is related to $u$ and, in turn, $e$. It follows that $\operatorname{cov}(z, e) \neq 0$, indicating the violation of the exclusion restriction. If $z$ is simultaneously determined with $x$, it is necessarily related to the error term in the outcome equation and cannot serve as a valid instrument. As spatial instruments are by design simultaneously determined with, and therefore a function of, the endogenous variable, they violate the exclusion restriction.

Note what this implies. First, a spatial instrument is exogenous to the outcome equation if $x$ is exogenous-that is, when no instrument would have been required. Conversely, the endogeneity of the instrument increases in the endogeneity of the variable of interest, and hence is most severe where the instrument is needed most. Second, a spatial instrument is exogenous if there are no simultaneous relationships between units-that is, when the instrument is irrelevant. Thus, the strength of the instrument is, with spatial instruments, no longer independent from the exclusion restriction. When used as instrument for an endogenous variable, a spatial instrument cannot jointly satisfy both conditions required for a valid instrument: if the instrument is strong, it violates the exclusion restriction; if the instrument does not violate the exclusion restriction, it is irrelevant.

\section{Discussion}

We highlighted a simple problem: faced with an endogenous variable, using realizations of that variable in other units as instruments cannot produce unbiased, consistent estimates. First, these spatial instruments require arguing for one type of spatial dependence in the data, while simultaneously ruling out other-often plausible-spatial relationships. Second, by design,

7 In the presence of simultaneous spatial dependence, time-lagged realizations of the spatial lag are also invalid instruments. First, they do not avoid concerns of outcome interdependence or predictor spillovers. Second, to have power in the first stage, the time-lagged spatial lag is also necessarily related to the contemporaneous spatial lag-either directly through autocorrelation or indirectly via cross-unit, over-time relationships in $x$. In short, the relationships that give this instrument power also ensure it violates the exclusion restriction. Finally, including both the contemporaneous and time-lagged spatial lag inherits the problems discussed above (as the contemporaneous spatial lag is still an instrument) and, if estimating the first stage nonlinearly (as in any spatial-autoregressive model), this approach does not inherit the properties of 2 SLS.

$8 x_{j}$, the $j$ th element of $\mathbf{x}$ on the left-hand side of equation (6), is a function of $x_{i}$ and therefore $u_{i}$ on the right-hand side of equation (6). The simultaneity bias also produces a biased first-stage coefficient estimate $\gamma$ and, if any other spatial relationships remain unaccounted for, typically inflated estimates of instrument strength. The potentially undetected weak instrument compounds the simultaneity problem discussed here. 
these instruments violate the exclusion restriction, because the endogenous variable and the instrument are simultaneously determined. Despite the popularity of spatial instruments in the social sciences, these problems are rarely acknowledged, let alone addressed. Jointly, these two problems imply that spatial instruments cannot be valid instruments. They do not even guarantee an improvement over OLS. We therefore advocate against the use of spatial instruments in their current form, and caution against interpreting estimates based on those instruments as superior to OLS.

More generally, this letter points to a potential misunderstanding in some applications of instrumental variables. Two requirements are typically mentioned for valid instruments: the exclusion restriction and correlation with the endogenous variable. These are often jointly summarized as the requirement that the instrument affects the outcome through and only through the endogenous predictor. As Angrist, Imbens, and Rubin (1996, p. 450) emphasize, such language obfuscates the conditions necessary for valid instruments and "hinders assessment and communication of the plausibility of the underlying model." As causal diagrams as well as the potential outcome framework clarify, the exclusion restriction is more nuanced: there must be no pathway from the instrument to the outcome except through the variable of interest, and there must be no pathway to the instrument. Both are implied by the common assumptions for valid instruments, but the latter is often neglected in applications. Spatial instruments violate this condition and therefore cannot produce valid inferences.

\section{Supplementary material}

For supplementary material accompanying this paper, please visit

https://doi.org/10.1017/pan.2018.10.

\section{References}

Acemoglu, Daron, Simon Johnson, James A. Robinson, and Pierre Yared. 2008. Income and democracy. The American Economic Review 98(3):808-842.

Acemoglu, Daron, Suresh Naidu, Pascual Restrepo, and James A. Robinson. Forthcoming. Democracy does cause growth. Journal of Political Economy, to appear.

Angrist, Joshua D., Guido W. Imbens, and Donald B. Rubin. 1996. Identification of causal effects using instrumental variables. Journal of the American Statistical Association 91(434):444-455.

Ansell, Ben W. 2008. Traders, teachers, and tyrants: democracy, globalization, and public investment in education. International Organization 62(02):289-322.

Betz, Timm, Scott Cook, and Florian Hollenbach. 2017. Replication data for: on the use and abuse of spatial instruments, doi:10.7910/DVN/W4TXDU, Harvard Dataverse, V1.

Boix, Carles. 2011. Democracy, development, and the international system. American Political Science Review 105(04):809-828.

Büthe, Tim, and Helen V. Milner. 2008. The politics of foreign direct investment into developing countries: increasing FDI through international trade agreements? American Journal of Political Science 52(4):741-762.

Franzese, Robert J. Jr., and Jude C. Hays. 2007. Models of cross-sectional interdependence in political science panel and time-series-cross-section data. Political Analysis 15(2):140-164.

Gleditsch, Kristian Skrede, and Michael D. Ward. 2006. Diffusion and the international context of democratization. International Organization 60(4):911-933.

Kayser, Mark Andreas, and Michael Peress. 2012. Benchmarking across borders: electoral accountability and the necessity of comparison. American Political Science Review 106(3):661-684.

Manski, Charles F. 1993. Identification of endogenous social effects: the reflection problem. The Review of Economic Studies 60(3):531-542.

Persson, Torsten, and Guido Tabellini. 2009. Democratic capital: the nexus of political and economic change. American Economic Journal: Macroeconomics 1(2):88-126.

Sovey, Allison J., and Donald P. Green. 2011. Instrumental variables estimation in political science: a readers' guide. American Journal of Political Science 55(1):188-200.

Stasavage, David. 2005. Democracy and education spending in Africa. American Journal of Political Science 49(2):343-358. 